\title{
Mathematical model of the problem of accounting for losses in electrical networks when optimizing the loads of electrical consumers
}

\author{
Tulkin Gayibov', Kamal Reymov², Arislan Aytbaev² \\ ${ }^{1}$ Tashkent State Technical University, Tashkent, Uzbekistan, ${ }^{2}$ Karakalpak State University, Nukus, Uzbekistan,
}

\begin{abstract}
Based on the improvement of existing algorithms, an effective algorithm and program for taking into account the network factor have been developed for optimal planning of short-term modes of power systems with control of the load of power consumers, based on the restructuring of the dependences of the relative gains of losses on the power of nodes with settlement stations and regulated power consumers. As a result, it is possible to determine the optimal short-term modes of power systems, taking into account the network factor in terms of load management of power consumers.
\end{abstract}

\section{Introduction}

Taking into account losses in electrical networks and the corresponding network factor in the optimal planning of short-term modes of power systems with control of the load of electrical consumers, in the general case, can lead to a significant change in the load schedules of regulated electrical consumers and the redistribution of the total load of the power system between the calculated power plants. Here, optimization is carried out by reducing the total fuel costs in the power system by reducing losses as a result of choosing the optimal load schedules for power consumers and power plants participating in the optimization [1-2].

The problem under consideration is mathematically formulated as follows:

to minimize the total fuel costs at the calculated thermal power plants for the regulation cycle $\mathrm{T}$ :

$$
B=\sum_{t \in T} \sum_{i \in N} B_{i}\left(P_{i}^{t}\right)
$$

subject to restrictions:

- by the balance of capacities in the power system in each interval of the regulation cycle $\mathrm{T}$

$$
W_{t}=\sum_{i \in N} P_{i}^{t}-\sum_{j \in M} P_{j}^{t}-\pi_{t}=0, \quad t \in T,
$$

- according to the balance of electricity for a control cycle for each of the calculated electricity consumers

$$
\varphi_{j}=\sum_{t \in T} P_{j}^{t}-\ni_{j}^{T}=0, \quad j \in M,
$$

- by the limiting capacities of design power plants and electricity consumers

$$
P_{i}^{t, \text { min }} \leq P_{i}^{t} \leq P_{i}^{t, \max }, \quad i \in N, \quad t \in T,
$$

$$
P_{j}^{t, \text { min }} \leq P_{j}^{t} \leq P_{j}^{t, \max }, \quad j \in M, \quad t \in T,
$$

- on power flows in controlled power lines

$$
P_{l}^{t} \leq \bar{P}_{l}^{t}, \quad l \in L_{p}, \quad t \in T
$$

where N, M is a set TPS and power consumers involved in optimization; $L_{p}$ - lots of $\mathrm{P}_{\mathrm{L}}$, in which power flows are controlled; $P_{i}^{t}, P_{j}^{t}$ - power $\mathrm{i}$ - th TPS and $\mathrm{j}$ - th electric consumer in $\mathrm{t}-\mathrm{M}$ regulation cycle interval; - total losses of active power in electrical networks в $\mathrm{t}-\mathrm{m}$ regulation cycle interval; $B_{i}\left(P_{i}^{t}\right)$ fuel cost $\mathrm{i}-$ th THES at its load in $\mathrm{t}-\mathrm{M}$ regulation cycle interval $P_{i}^{t} ; W_{t}, \varphi_{j}$ functions of active power imbalances in $\mathrm{t}-\mathrm{M}$ interval and electricity $j$ - th consumer for the regulation cycle $\mathrm{T}$, respectively [3-4].

The described problem can be reduced to minimization of the following function

$$
L=B+\sum_{t \in T} \mu_{t}\left(\sum_{i \in N} P_{i}^{t}-\sum_{j \in M} P_{j}^{t}-\pi_{t}\right)+\sum_{j \in M} \lambda_{j}\left(\sum_{t \in T} P_{j}^{t}-\Im_{j}^{T}\right)+\sum_{t \in T} \sum_{l \in L_{p}} W_{l}^{t}
$$

where $\mu_{t}, \lambda_{j}$ - indefinite Lagrange multipliers to take into account the limitation in the form of the balance of active power in the power system in $\mathrm{t}-\mathrm{M}$ regulation cycle interval (2) and electricity for $\mathrm{j}-$ th consumer for a cycle of regulation (3); $W_{l}^{t}$ - penalty function introduced when the flow restriction is violated $1-$ th $\mathrm{PL}$ in $\mathrm{t}-\mathrm{m}$ regulation cycle interval. In the described mathematical model of the problem, it is assumed that the calculated HPS are taken into account based on the selection of the corresponding Lagrange multipliers $\lambda$ and their reduction, in the calculated sense, into the category of equivalent TPS as in [1-4]. And TPS with a limited fuel supply for the planned period are taken into account by the algorithm proposed in Chapter 3 of this work.

Thus, the mathematical description of the problem of 
optimal planning of the short-term mode of the power system with control of the load of electrical consumers and taking into account losses is distinguished by the introduction of the total losses of active power in electrical networks into the equation of the balance of active power for every t-rd time interval of the control cycle. These losses are complex functions of all operating parameters and, therefore, must be determined on the basis of calculating the steady-state modes of electrical networks for all intervals of the regulation cycle.

By analogy with the optimal planning of the short-term operation of the power system without controlling the load of power consumers, the problem under consideration can be solved on the basis of two approaches.

In the first, the optimal planning of the short-term mode of the power system with the optimization of the loads of the regulated electric consumers is carried out immediately for the entire period $\mathrm{T}$. This planning can be carried out, in particular, by minimizing the function (7) with a suitable optimization method. Due to the large dimension of the resulting problem and the presence in it, in the general case, of a large number of different-scale parameters of different physical nature, the iterative calculation process often has an oscillatory character and may diverge. In addition, solving the problem based on this approach requires performing a large amount of computational operations at each iteration.

In the second approach, the problem is solved on the basis of dividing the regulation cycle into calculated intervals. In this case, the optimization is performed sequentially for each interval of the control cycle.

Due to the presence of a large number of integral constraints, which are the conditions for the balance of electricity for each of the regulated electric consumers, the accounting of which in the case of separate optimization for individual intervals is a difficult task, the first approach is used in the algorithms for solving this problem proposed below. Below are the proposed algorithms for solving the problem under consideration.

\section{The mathematical statement of the problem}

At optimal values of the variables - the active powers of the design power plants and loads with controlled electric consumers, the condition is written by the method of indefinite Lagrange multipliers:

$$
\left.\begin{array}{l}
\frac{\partial L}{\partial P_{i}^{t}}=b_{i}^{t}+\mu_{t}\left(1-\sigma_{i}^{t}\right)+\sum_{l \in L_{p}} u_{l i}^{t}=0, \quad i \in N, t \in T ; \\
\frac{\partial L}{\partial P_{j}^{t}}=-\mu_{t}\left(-1-\sigma_{j}^{t}\right)+\lambda_{j}+\sum_{l \in L_{p}} m_{l j}^{t}=0, \quad j \in M, t \in T ; \\
\frac{\partial L}{\partial \mu_{t}}=W_{t}=0, \quad t \in T ; \\
\frac{\partial L}{\partial \lambda_{j}}=\varphi_{j}=0, \quad j \in M,
\end{array}\right\}
$$

Where $b_{i}^{t}=\frac{\partial B_{i}}{\partial P_{i}^{t}}$-the relative increase in the consumption of equivalent fuel per i-TPS under load t- interval $P_{i}^{t}$; $w_{l i}^{t}=\frac{\partial W_{l}}{\partial P_{i}^{t}}, \quad w_{l j}^{t}=\frac{\partial W_{l}}{\partial P_{j}^{t}}$-derivatives of the penalty function to take into account the power flow restriction 1monitored power line i- TPS $P_{i}^{t}$ and j-design load $P_{j}^{t}$ in t$\mathrm{m}$ interval of the planning period $\mathrm{T} ; \sigma_{i}^{t}=\frac{\partial \pi_{t}}{\partial P_{i}^{t}}$ - the derivative of the total loss of active power in electrical networks in $\mathrm{t}-\mathrm{m}$ interval of the power control cycle i- TPS; $\sigma_{j}^{t}=\frac{\partial \pi_{t}}{\partial P_{j}^{t}}$ - the derivative of the total loss of active power in electrical networks in $\mathrm{t}-\mathrm{m}$ interval of the power regulation cycle of the $\mathrm{j}$-th load node with regulated power consumers. Dividing the first equation into (8) on the $1-\sigma_{i}^{t}$ and the second equation for $1+\sigma_{j}^{t}$, we get one equation

$$
\frac{b_{i}^{t}+\sum_{l \in L_{p}} m_{l i}^{t}}{1-\sigma_{i}^{t}}=\frac{\lambda_{j}+\sum_{l \in L_{p}} m_{l j}^{t}}{1+\sigma_{j}^{t}},
$$

Designating the network power factors $\mathrm{i}$ - TPS in $\mathrm{t}-\mathrm{m}$ interval of the control cycle as $\eta_{i}^{t}=\frac{1}{1-\sigma_{i}^{t}}$, by power $\mathrm{j}$-load node in t- $\mathrm{m}$ interval of the control cycle as $\eta_{i}^{t}=\frac{1}{1+\sigma_{j}^{t}}$, system of equations (4.8) represent in the following form:

$$
\begin{aligned}
& \eta_{i}^{t}\left(b_{i}^{t}+\sum_{l \in L_{p}} u_{l i}^{t}\right)=\eta_{j}^{t}\left(\lambda_{j}+\sum_{l \in L_{p}} u_{l j}^{t}\right), \quad t \in T(i \in N, j \in M) ; \\
& \sum_{i \in N} P_{i}^{t}-\sum_{j \in M} P_{j}^{t}-\pi_{t}=0, \quad t \in T \\
& \sum_{t \in T} P_{j}^{t}-Э_{j}^{T}=0, \quad j \in M .
\end{aligned}
$$

The latter system, together with simple constraints (4) and (5) is a condition for the optimal coverage of the load schedule of the power system by thermal power plants, taking into account losses in electrical networks with optimal control of the load of regulated power consumers.

The solution of the problem under consideration with the known total losses of active power and their derivatives for all intervals of the control cycle, in principle, can be carried out on the basis of solving the system of equations obtained from (9). However, in this case, in the general case, the convergence of the iterative process is unreliable. He hesitates and may diverge. Therefore, the resulting system is mainly used to represent the essence of the considered optimization problem, taking into account losses in electrical networks [5-6].

In the proposed algorithm, the problem of optimal planning of a short-term mode of a power system with optimization of loads of electric consumers and taking into; 
account losses in networks is solved by minimizing the function

$$
F=B+\sum_{t \in T} \sum_{l \in L_{p}} W_{l}^{t}
$$

subject to all the specified restrictions using the gradient method. In this case, to take into account the restrictions (2) and (3) the optimized capacities of settlement stations and load nodes with regulated power consumers are divided into two groups - independent and dependent variables. Constraints imposed on independent variables are taken into account by fixing at each iteration the variables that have gone beyond the permissible limits at the violated limit values with checking the need to detach them in subsequent iterations.

Since the total losses of active power in electrical networks and their derivatives are complex functions of all operating parameters, their values are refined iteratively based on the calculation of the steady-state modes of electrical networks. In this case, to take into account the restrictions (2) and (3) the optimized capacities of settlement stations and load nodes with regulated power consumers are divided into two groups - independent and dependent variables. The imposed constraints on the independent variables are taken into account by simply fixing at each iteration the variables that have gone beyond the permissible limits at the violated limit values, checking the need to detach them in subsequent iterations.

Thus, the total losses of active power in electrical networks and their derivatives are complex functions of all operating parameters, their values are refined iteratively based on the calculation of the steady-state modes of electrical networks.

In connection with the computational difficulties of taking into account integral constraints, a new algorithm for optimal planning of the short-term mode of the power system with control of the load of power consumers and taking into account losses in the networks is proposed, based on restructuring the power characteristics of stations for the entire planning period, taking into account the network factor. The essence of the algorithm is as follows:

1) optimal coverage of the total load schedule of the power system is carried out by all design TPSs with optimal control of the load of electrical consumers without taking into account losses in electrical networks. As a result, optimal load graphs of design power plants and power consumers are obtained;

2) for each interval of the regulation cycle, calculations of the steady-state modes of electrical systems are performed, the total losses in electrical networks, their derivatives and network coefficients for the capacity of stations and load nodes are determined. Due to the unevenness of the load schedules of stations and consumers, dependencies are obtained here $\sigma_{i}\left(P_{i}\right)$ and $\sigma_{j}\left(P_{j}\right)$;

3 ) multiplying the characteristic of relative gains $(\mathrm{CRG})$ of the settlement stations by the corresponding dependencies $\eta_{i}\left(P_{i}\right)$ get CRG taking into account the network factor $\bar{b}_{i}\left(P_{i}\right)=\eta_{i}\left(P_{i}\right) \times b_{i}\left(P_{i}\right)$;

4) optimal planning of the power system mode for the period under consideration is carried out using the rebuilt
CRG settlement stations $\bar{b}_{i}\left(P_{i}\right)$ as without taking into account the network factor. At this stage, when calculating the components of the gradient according to the loads of nodes with controlled consumers, the obtained as a result of performing Sec. 2 dependencies $\sigma_{j}\left(P_{j}\right)$.

\section{Conclusions}

1. The conditions for the optimal coverage of the power system load schedule with optimal control of the load of regulated power consumers, taking into account the losses of active power and the network factor, are obtained.

2. A mathematical model is proposed for the problem of optimal planning of a short-term mode of the power system with optimal control of the load of electric consumers, as well as taking into account the losses of active power and the network factor.

\section{References}

1. T Gayibov, B Pulatov, Sh Latipov, and Gulnaz Turmanova. Power System Optimization in Terms of Uncertainty of Initial Information. E3S Web of Conferences139,01031(2019) RSES2019. https://doi.org/10.1051/e3sconf/2019130

2. T Gayibov, B Pulatov. Optimization of Short-term Modes of Hydrothermal Power System. E3S Web of Conferences 209, 07014 (2020) ENERGY-21. https://doi.org/10.1051/e3sconf/202020907014

3. Tulkin Gayibov, Sherxon Latipov, and Bakhadir Uzakov. Power System Mode optimization by piecewiselinear approximation of energy characteristics of Power Plants. E3S Web of Conferences 139, (2019) RSES 2019. https://doi.org/10.1051/e3sconf/20191390

4. T Gayibov, Sh Latipov, D Abdurashidov, B Pulatov and A Davirov. Algorithm for power systems mode optimization taking into account the frequency change in terms of probablistic nature of initial information. IOP Conf. Series: Materials Science and Engineering 883 (2020) 012185 IOP Publishing doi:10.1088/1757$899 X / 883 / 1 / 012185$

5. Reymov, K.M., Turmanova, G.M., Makhmuthonov, S.K., Uzakov, B.A.//Mathematical models and algorithms of optimal load management of electrical consumers. E3S Web of Conferences, 2020, 216, 01166. https://doi.org/10.1051/e3sconf/202021601166

6. Reymov, K.M., Rafikova, G.R., Nematov, L., Esemuratova, S. //Existing Condition and Prospects of Making Power Balance and Managing Load of Electric Consumers in Uzbek Power System. E3S Web of Conferences, 2020, 209, 07015. https://doi.org/10.1051/e3sconf/202020907015 